\title{
Performance analysis of CIC multirate filter in cognitive radio for efficient pulse shaping in wireless domain
}

\author{
A. S. Kang, Vishal Sharma, Renu Vig \\ Department of ECE, UIET, Panjab University, India
}

\begin{tabular}{l}
\hline \hline Article Info \\
\hline Article history: \\
Received May 5, 2018 \\
Revised Mar 6, 2019 \\
Accepted Nov 13, 2019 \\
\hline
\end{tabular}

\section{Keywords:}

CIC

Cognitive radio

Filter

Multicarrier

\begin{abstract}
In this paper, the Performance Analysis of Cascade Integrator Comb Filter in context of Filter Bank Multicarrier Transmission has been presented for Cognitive radio. A benefit of the chosen technique is that, a CIC filter can be designed with a slight adjustment in parameters of interest. The entire performance of the filters designed is analyzed and evaluated by analyzing Normalized Amplitude versus Normalized Frequency plots at typical $\mathrm{K}$ and $\mathrm{N}$ values. The roll off factor plays a significant role in performance analysis of CIC filter. The results shown are a useful advance for rf design engineers working in the domain of multirate signal processing in wireless communication. To ensure the acceptable performance of Enhanced FBMC, computational complexity, and transmission burst length need to be reduced. The effect of Stop band attenuation on the edges of Magnitude and Frequency responses has been studied under constraints such as Lp, $\mathrm{K}$ and $\mathrm{M}$ during different simulation runs.
\end{abstract}

Copyright $(0) 2019$ Institute of Advanced Engineering and Science. All rights reserved.

\section{Corresponding Author:}

\author{
A. S. Kang, \\ Department of ECE, UIET, \\ Panjab University, Chandigarh, India. \\ Email: askang_85@yahoo.co.in
}

\section{INTRODUCTION}

Different pulse shapes and various other performance parameters of interest such as filter length, delay, hardware computational complexity and cost etc which affects the performance efficiency of FBMC Multicarrier Communication due to increased spectral efficiency and reduced distortion effect with higher stopband attenuation possibly achievable under different persisting constraints of overlapping factor K, number of frequency channels $\mathrm{M}$, with suitable encoding and modulation technique applied therein to attain Perfect Signal Reconstruction, with ultimately fulfiling the Nyquist criteria of efficient pulse shaping. An interesting CIC Filter analysis is appropriate for rf wireless system design engineers to pave the way for the success of next generation wireless communication systems.

More Spectral Resolution, Reduction in out of band emission and enhanced bit rate because of lesser guard bands with no cyclic prefix makes FBMC to assign different subcarriers to available unsynchronized users in a dynamic manner [1]. Fiter Bank Multicarrier relies upon a prototype filter with good time-frequency product. The confinement of FBMC signal in both time and frequency domain tends to increase its robustness to timing error and offset in frequency. A unique algorithm with unified structure for FBMC Transceiver has been proposed [1].The capability of FBMC to provide improvement in frequency selectivity by making use of longer and spectrally efficient filter makes TMUX design which comprises Analysis (Downsampling) and Synthesis Filter Bank(Upsampling).The rise and fall in the signal sampling rate is related to the complex to real and real to complex conversion of input signal, with pre and post OQAM sub channel processing. The study related to the FBMC subchannel processing, depends upon complicated filter design process that corresponds to varying filter lengths, has been shown here. The Overlapping factor, $\mathrm{K}$ taken is in the suitable 
reference range (2-20) [2]. It defines the maximum number of multicarrier symbols which overlap each other in time domain.

The prototype filter is analyzed to fulfill the Near Perfect Reconstruction characteristics. The Perfect Reconstruction is only achieved in case of ideal transmission channel which implies that interferences originating from filter bank are too small when compared to the residual interferences due to transmission channel. The Cascaded Interconnection prototype filters could provide highest stopband attenuation than their equal length PR counterparts. The best way to design the NPR prototype filter is to directly optimize the coefficients of the filter impulse response. The only limitation of this technique is that the number of filter coefficients increase sharply when filter bank is designed with number of subchannels higher than 256 [3, 4]. The overlapping factor $\mathrm{K}=4$ and localized mode band allocation of $\mathrm{Ms}=160$ subcarriers has been used in the core of the FBMC system [5].

\section{COGNITIVE RADIO NETWORK}

FBMC Transceiver for multi user asynchronous transmission on the fragmented spectrum plays a major role in spectrum sensing in wireless cognitive radio network though it also depends upon the challenges and design trade-offs which are required. The intelligence in wireless cognitive radio network is introduced by spectrum sensing the bandwidth size on multiple channels with varying signal strengths and different transmission techniques $[6,7]$. Good spectral containment is mandatory for getting rid of the distortion effect from asynchronous signals in adjacent bands. There is always a requirement to minimize the delay of prototype filter as the selection of number of coefficients is a trade-off between delay and filter stop band attenuation $[8,9]$.

\subsection{Multi mode transmission in filter bank processing}

The Filter Bank Processing is based on multiple access technique which enables the mobile terminals to transmit at a time in reverse link at different operation modes, namely FBMC, FB-FBMC and also in serial carrier modulation just to provide independent uplink signal multiplexes with more bandwidth efficiency. The reverse link can support both single carrier and multicarrier modes by utilising the better selectivity of frequency sampling-based prototype filter design. A lower calculation complexity based linear equalizer structure, is capable of frequency selective per sub band processing to facilitate efficient channel compensation [10]. Infact, the optimization of Modified DFT Filter Bank Multicarrier Modulation System is very useful for the design of modulation systems in the next generation networks [11, 12].

\section{FREQUENCY SYNCHRONIZATION FOR FBMC TRANSMISSION}

The higher stopband attenuation of the Filter bank allows channel selection filtering and Narrowband Interference reduction at the receiver analysis bank, without pre-processing, besides the anti-aliasing filtering determined by the sampling rate at analysis filter bank. Hence, the Filter Bank provides good frequency selection for the desired spectral components, so it is desirable to think of a FBMC receiver where all the baseband signal processing functions are performed in frequency domain. The entire channel parameter estimation is done after analysis filter bank at a lesser rate [13]. Practically, the transmission systems are peak power limited as they show non-linear characteristics which cause spectral widening of the transmit signal. The current communication systems need to restrict the power spectral density of the transmit signal to correctly specified spectrum mask $[14,15]$

\section{ALGORITHM}

The design of Cascaded Integrator Comb Filter-Prototype Filter for plotting Amplitude w.r.t Normalized Frequency Response has been shown here to examine the effect of different parameters.

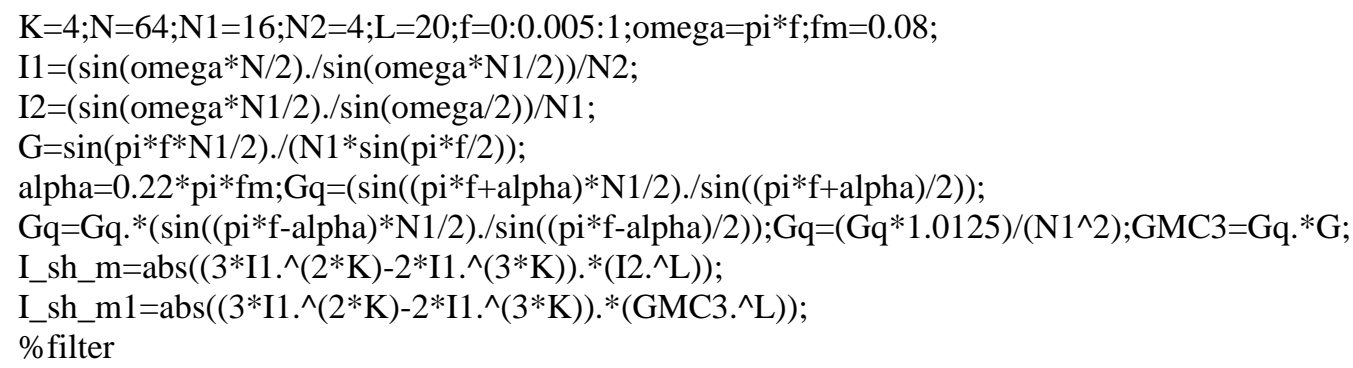


Figure (2)

$\operatorname{plot}(\mathrm{f}, 20 * \log 10($ I_sh_m $), f, 20 * \log 10($ I_sh_m1))

axis $([0,0.2,-500,5])$; grid on; hold on;

$\mathrm{G}=\left(\sin (\right.$ omega* $\mathrm{N} / 2) . / \sin ($ omega/2) $) / \mathrm{N} ; \mathrm{GK}=\mathrm{G} .{ }^{\wedge} \mathrm{K}$;

Figure(2)plot(f,20*log10(abs(GK)),'k','LineWidth',2)

I_sh=abs( $3 *$ G.^(2*K)-2*G.^(3*K));plot(f,20*log10(I_sh),'--')

\section{RESULTS AND DISCUSSION}

Normalised amplitude versus normalised subcarrier frequency plot between K and Alpha as shown in Figure 1-3. Computation of normalized amplitude, stopband attenuation occuring at variable overlapping factor $\mathrm{K}$ and roll off factor as shown in Table 1. Various Matlab Plots obtained between K, Alpha and Stopband Attenuation for CIC Prototype Filter Design have been shown in Figure 4-11 depict the analytical comparison done.

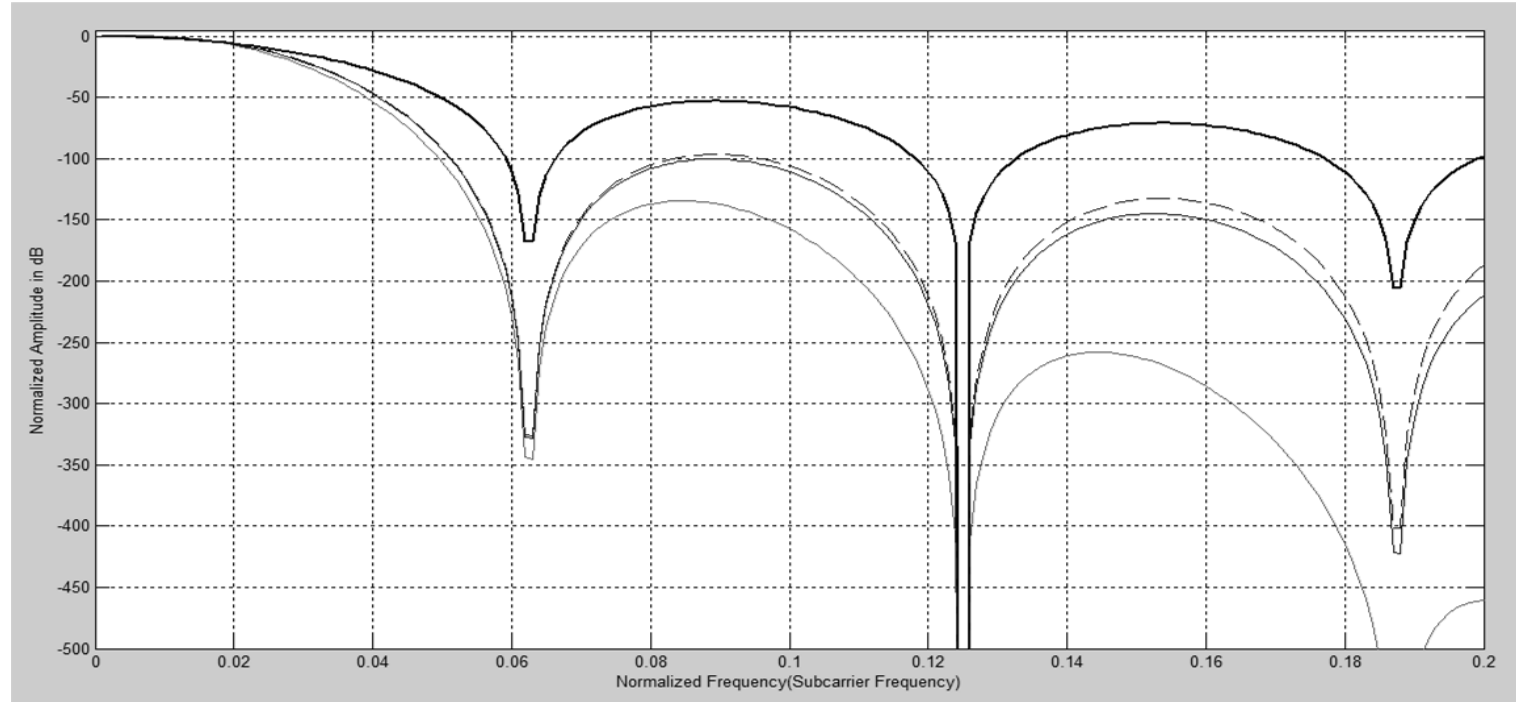

Figure 1. Normalised amplitude versus normalised subcarrier frequency plot at $K=4$, Alpha $=0.22$

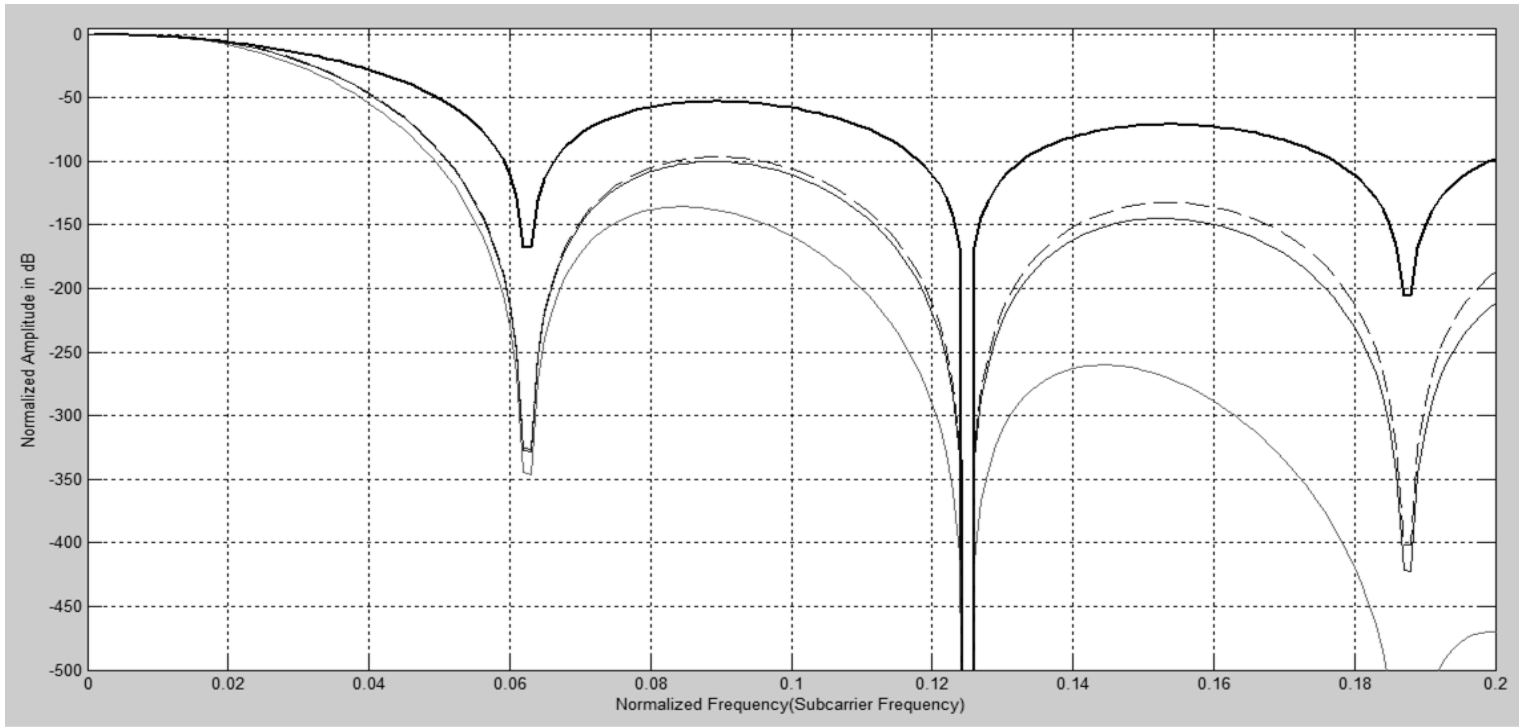

Figure 2. Normalized amplitude versus normalized subcarrier frequency Plot at $K=4$, Alpha $=0.78$ 


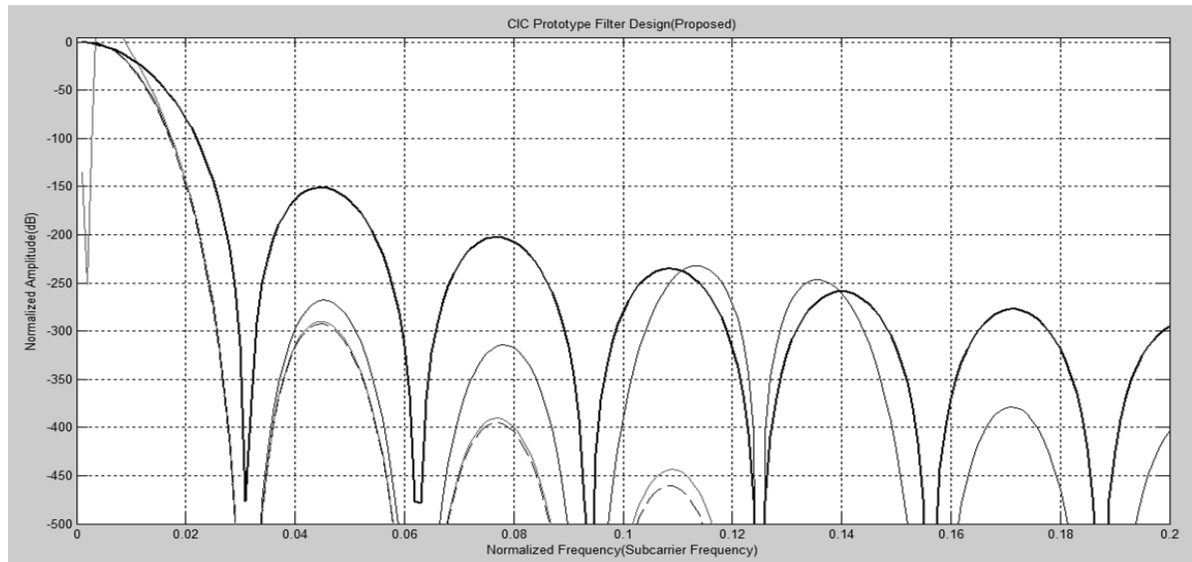

Figure 3. Normalised amplitude versus normalized frequuency plot for CIC proposed prototype at K=11.4,Alpha=0.22

Table 1. Computation of normalized amplitude(dB), stopband attenuation occuring at variable overlapping factor $\mathrm{K}$ and roll off factor

\begin{tabular}{|c|c|c|c|}
\hline $\begin{array}{l}\text { Overlapping Factor } \\
\mathrm{K}\end{array}$ & $\begin{array}{l}\text { Roll off factor } \\
\text { Alpha }\end{array}$ & $\begin{array}{c}\text { Normalized } \\
\text { Amplitude }(\mathrm{dB})\end{array}$ & $\begin{array}{c}\text { Stopband Attenuation starting at a Normalized } \\
\text { Frequency }\end{array}$ \\
\hline 4 & 0.22 & -10 to $-341 \mathrm{db}$ & 0.0682 (Normal Trend) \\
\hline 4 & 0.78 & -10 to $-348 \mathrm{db}$ & 0.0691 Early side lobe tail decay \\
\hline 11.4 & 0.22 & -10 to $-462 \mathrm{db}$ & 0.0352(Faster Side lobe tail decay) \\
\hline
\end{tabular}

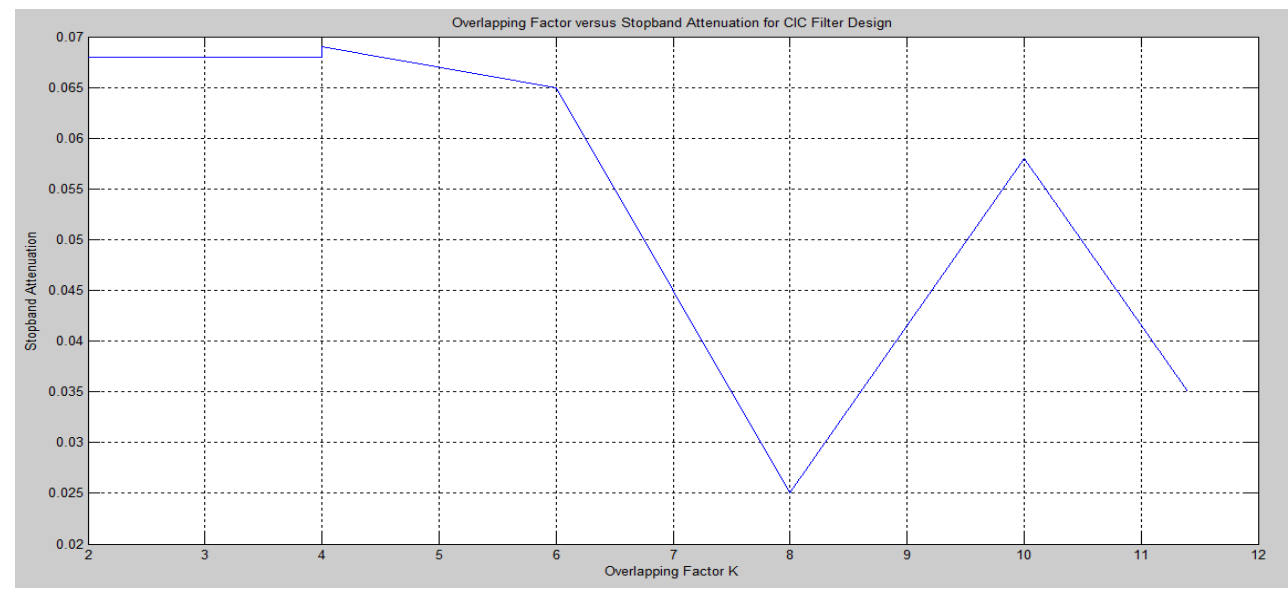

Figure 4. Overlapping factor K versus stopband attenuation for CIC filter design

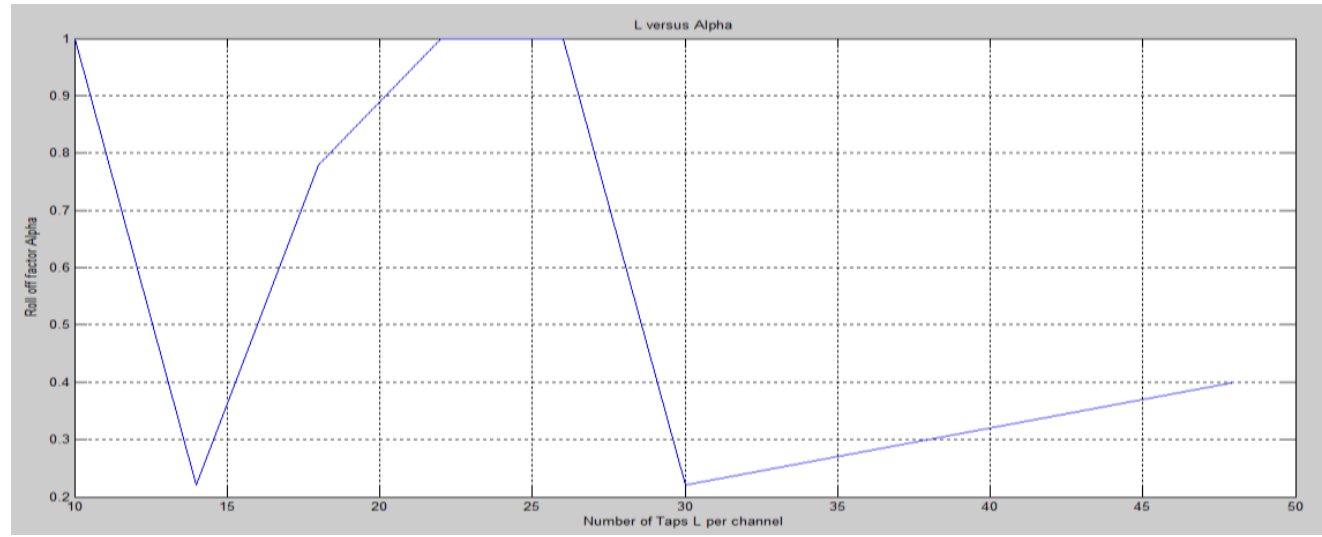

Figure 5. Number of taps per channel versus roll off factor alpha for CIC filter design 


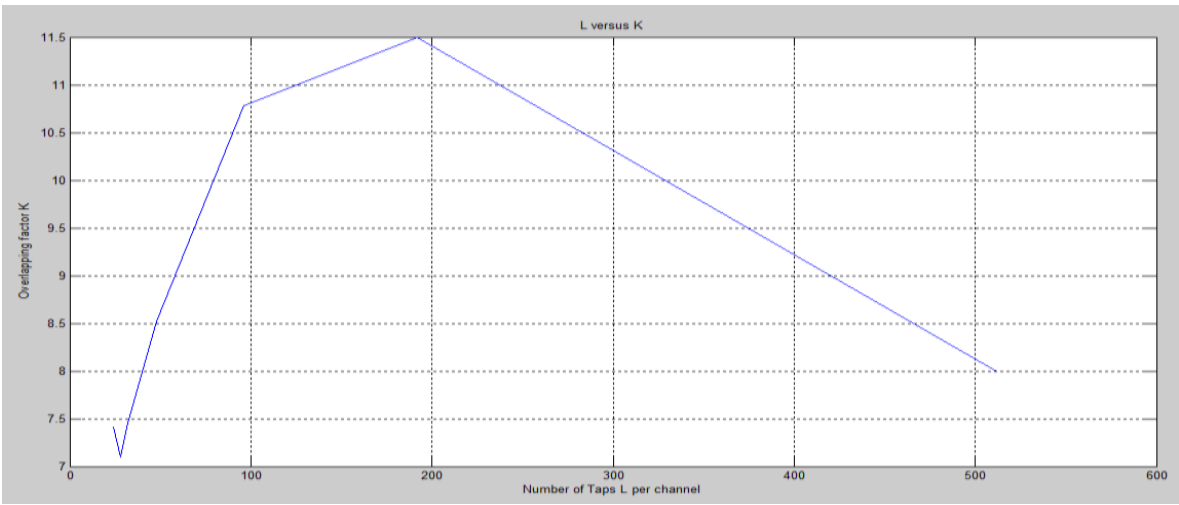

Figure 6. Number of taps L per channel versus overlapping factor K for CIC filter design

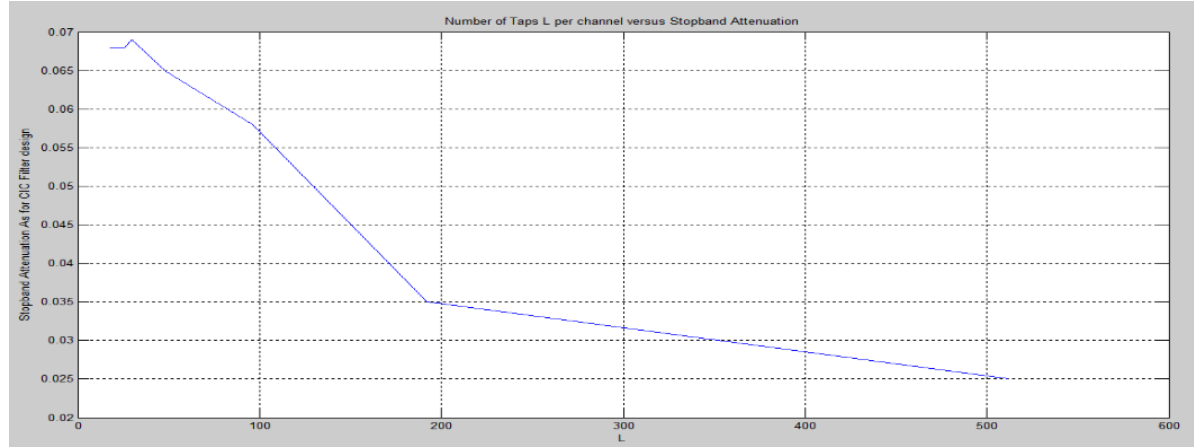

Figure 7. Number of taps L per channel versus stopband attenuation for CIC filter

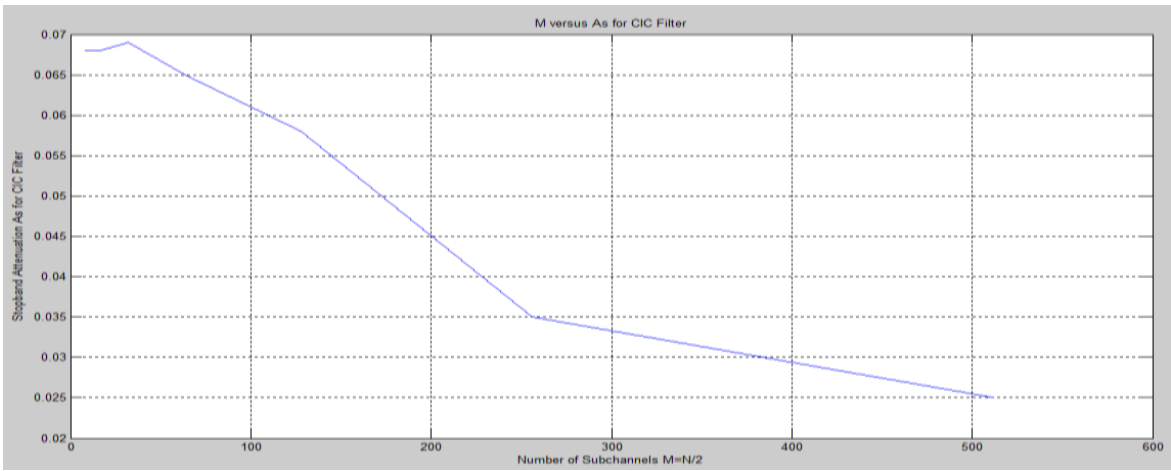

Figure 8. $M=N / 2$ versus stopband attenuation $A_{s}$ for CIC filter design

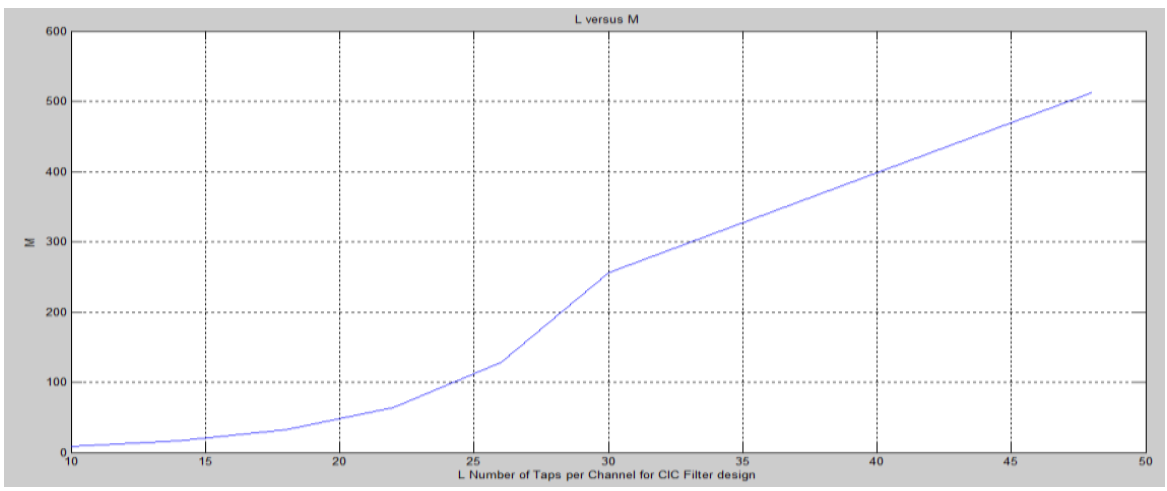

Figure 9. M number of Subchannels versus L number of taps per channel for CIC filter design 


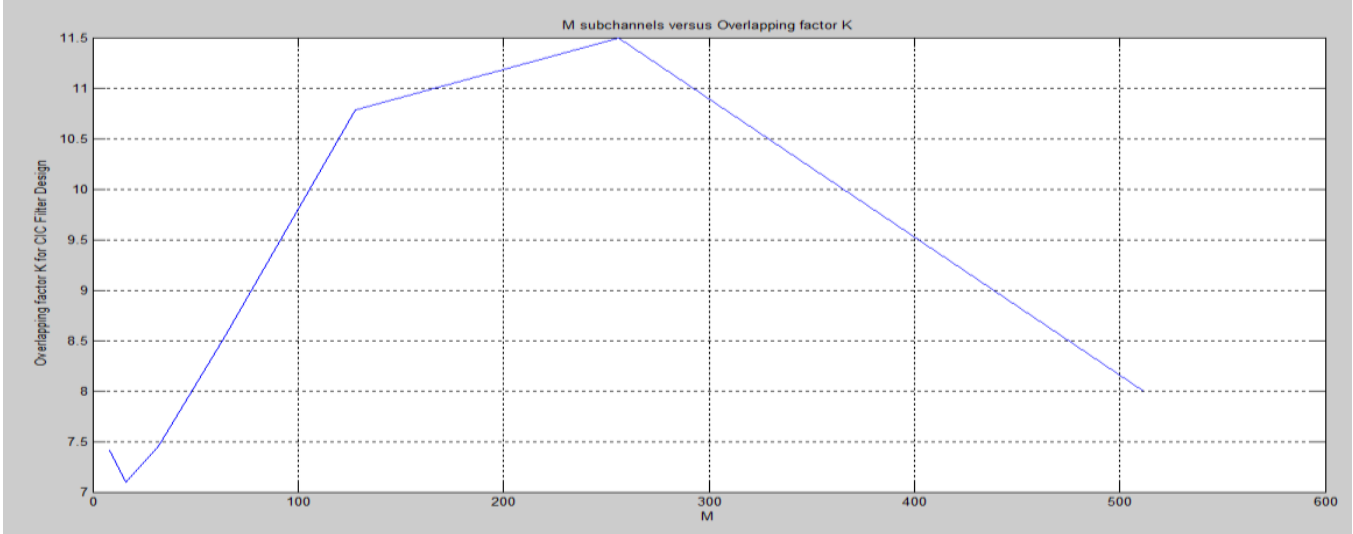

Figure 10. M subchannels versus overlapping factor K for CIC filter design

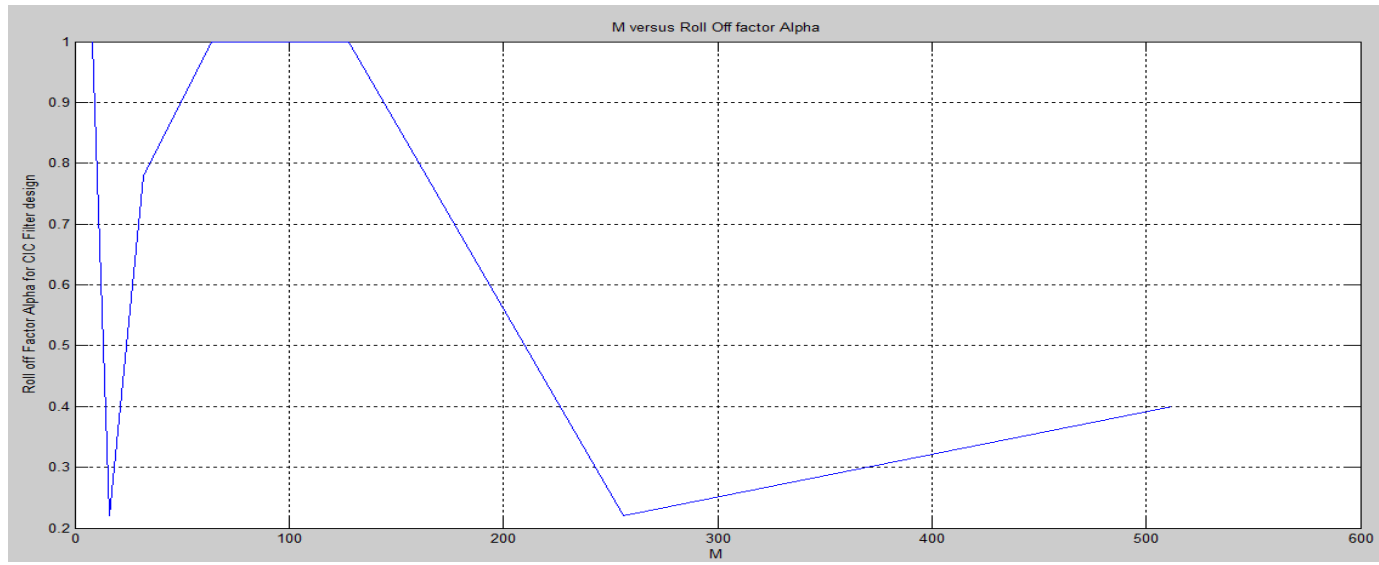

Figure 11. M subchannels versus roll off factor alpha for CIC filter design

\section{CONCLUSION}

The study done has its impact on the Performance Enhancement of Filter Bank Multicarrier System based Cognitive Radio under the effect of ubiquitous and pervasive radio communication. The results of proposed CIC Filter design are compared to those obtained by Wessel Lubberhuizen. It has been observed that $\mathrm{K}=8$ with Roll off factor=0.4 gives optimum results in terms of normalized amplitude of $-10 \mathrm{db}$ to beyond $500 \mathrm{db}$ with stopband attenuation occurring at a normalized frequency of 0.026 indicating faster side lobe tail decay happening irrespective of pulse shaping constraints. Different simulation runs have been done to obtain the results for CIC filter design in terms of Stopband Attenuation As, Overlapping Factor K, L taps per channel and Roll off factor Alpha $(0<\alpha<1)$.

\section{IMPACT OF STUDY AND FUTURE SCOPE}

The present work can be extended for further analysis on MAC layer protocol model using NS2 and adaptive signal processing approach, can be applied to highlight the effect of ambiguity surfaces of different prototype filters corresponding to different pulse shapes and various other performance parameters of interest such as filter length, delay, hardware computational complexity and cost etc which affects the performance efficiency of FBMC Multicarrier Communication due to increased spectral efficiency and reduced distortion effect with higher stopband attenuation possibly achievable under different persisting constraints of overlapping factor $\mathrm{K}$, number of frequency channels $\mathrm{M}$, with suitable encoding and modulation technique applied therein to achieve Perfect Reconstruction of the signal, with ultimate fulfilment of Nyquist criteria of efficient pulse shaping. An interesting CIC Filter analysis is appropriate for rf wireless system design engineers to pave the way for the success of next generation wireless communication systems [16, 17]. The analysis of CIC Multirate Filter in Cognitive Radio for Efficient Pulse Shaping has been done which shows its significance as reflected in the earlier work done for the ubiquitous-pervasive computing through matlab based simulations in the wireless domain [18-22].

Performance analysis of CIC multirate filter in cognitive radio for efficient pulse shaping ... (A. S. Kang) 


\section{ACKNOWLEDGEMENT}

The help rendered by Prof. Dr. Renu Vig is duly acknowledged. She was instrumental in guiding and implementing the work plan of the present research done. The authors are thankful to Research Promotion Cell, Panjab University Chandigarh for motivation.

\section{REFERENCES}

[1] Yun Cui, Zhifeng Zhao et al, "An Efficient Filter Banks Based Multicarrier System in Cognitive Radio Networks," Radio Engineering, vol. 19, no. 4, pp.479-487, 2010.

[2] PHYDYAS, "PHYDYAS-physical layer for dynamic spectrum access and cognitive radio," http:www.ictphydyas.org/2013.

[3] Maurice G Bellanger, "Specification and Design of a Prototype Filter for Filter Bank Based Multicarrier Transmission," Proc. IEEE International Conference, Paris, France, pp.2417-2420, 2001.

[4] Peiman Amini, Behrouz Farhang-Boroujeny, "A Comparison of Alternative Filter Bank Multicarrier Methods for Cognitive Radio Systems," Proc.Software Defined Radio Technical Conference, SDR 2006, pp.117-123, 2006.

[5] Damien Roque, Cyrille Siclet, "A Performance Comparison of FBMC Modulation Schemes with Short Perfect Reconstruction-Filters," Proc.19 ${ }^{\text {th }}$ International Conference on Telecommunications, ICT 2012, Lebanon, pages 6.

[6] Marius Caus, Ana I. Perez-Neira, "A Suboptimal Power Allocation Algorithm for FBMC/OQAM," Proc.IEEE International Conference on Acoustics, Speech and Signal Processing, ICASSP, pp.2660-2664, 2011.

[7] Y. Medjahdi, M. Terre, "Inter Cell Interference Analysis for OFDM/FBMC Systems," Proc.IEEE International Conference 2009, pp.598-602, 2009.

[8] Dirk S. Waldhauser Leonardo G. Baltar et al, "Comparison of Filter Bank based Multicarrier Systems with OFDM," Proc.IEEE International Conference, APCCAS 2006, pages 5, 2006.

[9] Tero Ihalainen, Ari Viholainen et al, "Filter Bank Based Multimode Multiple Access Scheme for Wireless Uplink," Proc. $17^{\text {th }}$ European Signal Processing Conference, Glasgow, Scotland, pp.1354-1358, 2009.

[10] Mohammed Abo-Zahhad, "Current state and Future Directions of Multirate Filter Banks and their applications," Digital Signal Processing, vol.13, pp.495-518, 2003.

[11] Alphan Sahin, Ismail Guvenc, "A Survey on Multicarrier Communications: Prototype Filters, Lattice Structures and Implementation Aspects," IEEE Communications Surveys \& Tutorials, vol. 16, no. 3, pp.1312-1338, 2014.

[12] Pilar Martin-Martin, Robert Bregovic et al, "A Generalized Window Approach for Designing Transmultiplexers," IEEE Transactions on Circuits Systems, II, vol. 54, no. 7, pp. 631-635, 2007.

[13] Eleftherios Kofidis and Dimitrios Katselis, "Improved Interference Approximation Method for Preamble Based Channel Estimation in FBMC/OQAM," Proc.19 $9^{\text {th }}$ European Signal Processing Conference, pp.1603-1607, 2011.

[14] Tzung Hwui Luo, Chih Hao Liu et al, "Design of Channel resilient DFT Bank Transceivers," IEEE Transactions on Signal Processing, pages 4, 2005.

[15] T. H. Stitz, Tero Ihalainen et al, "Practical Issues in Frequency Domain Synchronization for Filter Bank Based Multicarrier Transmission," Proc.IEEE International Conference ISCCSP 2008, pp.411-416, 2008.

[16] Guangyu Wang, Weiwei Zhang et al, "Optimization and Implementation for the Modified DFT Filter Bank Multicarrier Modulation System," Journal of Communications, vol. 8, no. 10, pp.651-657, 2013.

[17] [T. H. Stitz, Tero Ihalainen et al, "Mitigation of Narrowband Interference in Filter Bank Based Multicarrier Systems," Proc. IEEE Int Symp. Circuits and Systems, pages. 6, 2006.

[18] A. S. Kang, Vishal Sharma, Renu Vig, "Performance Evaluation of Prototype FBMC Cognitive Radio under constraints of L number of Sensing Samples at Variable SNR for AWGN and Rayleigh fading Environment," International Journal of Advanced Science \& Technology (IJAST), SERSC Australia, vol. 107, pp.1-20, 2017.

[19] A. S. Kang, Vishal Sharma, Renu Vig, "Performance Evaluation of OFDM and Prototype FBMC-OQAM Cognitive Radio under constraints of variable Overlapping factors," International Journal of Engineering Research \& Development, vol.13(9), pp.71-91, 2017.

[20] A. S. Kang, Renu Vig, "Design \& Implementation of Efficient Analysis and Synthesis QMF Bank for Multicarrier Cognitive Radio Communication," TELKOMNIKA-Telecommunication, Computing, Electronics \& Control, vol. 15, no. 2, pp. 636-645, 2017.

[21] A. S. Kang, Renu Vig, "Performance Analysis of Near Perfect Reconstruction Filter Bank in Cognitive Radio Environment," International Journal of Advanced Networking and Applications, vol.8(3), pp.3070-3083, 2016.

[22] A. S. Kang, Renu Vig, "Performance Analysis of FBMC-CR through Optimization -Tradeoff between Various Performance Parameters in the Cognitive Radio Environment," TELECOMMUNICATION SYSTEMS, Springer, vol. 63 , p. 23,2016 